\title{
Antidepressant-like effects of $\Delta 9$-tetrahydrocannabinol and rimonabant in the olfactory bulbectomised rat model of depression
}

\begin{abstract}
The endocannabinoid signalling system is widely accepted to play a role in controlling the affective state. Plant cannabinoids are well known to have behavioural effects in animals and humans and the cannabinoid CB1 receptor antagonist rimonabant has recently been shown to precipitate depression-like symptoms in clinical trial subjects. The aim of the present study was to investigate the behavioural and neurochemical effects of chronic administration of $q 9$ tetrahydrocannabinol (THC) and rimonabant on intact and olfactory bulbectomised (OB) rats used as a model of depression.

As expected, OB rats were hyperactive in the open field. Repeated THC ( $2 \mathrm{mg} / \mathrm{kg}$, i.p. once every $48 \mathrm{~h}$ for 21 days) and rimonabant ( $5 \mathrm{mg} / \mathrm{kg}$, i.p. once every $48 \mathrm{~h}$ for 21 days) reduced this hyperactivity, which is typical of clinically effective antidepressant drugs. In intact animals, chronic THC increased brain derived neurotrophic factor (BDNF) expression levels in the hippocampus and frontal cortex but rimonabant had no effect. Rimonabant increased the levels of phosphorylated extracellular signal regulated kinases (p-ERKs1/2) in the hippocampus and prefrontal cortex and THC also increased expression in frontal cortex. OB did not affect BDNF or p-ERK1/2 expression in the hippocampus or frontal cortex and in, contrast to the intact animals, neither THC nor rimonabant altered expression in the OB rats.

These findings indicate antidepressant-like behavioural properties of both THC and rimonabant in $\mathrm{OB}$ rats although additional studies are required to clarify the relationship between the chronic effects of cannabinoids in other pre-clinical models and in human depression.
\end{abstract}

Keyword: Cannabinoids; 9 -tetrahydrocannabinol; Rimonabant; Olfactory bulbectomy 